\title{
Measurement accuracy of Wi-Fi FTM on actual devices
}

\author{
Masakatsu Ogawa ${ }^{1, \text { a) }}$ and Hoyeon Choi ${ }^{1}$ \\ ${ }^{1}$ Faculty of Science and Technology, Sophia University, \\ 7-1 Kioi-cho, Chiyoda-ku, Tokyo 102-8554, Japan \\ a)m-ogawa@sophia.ac.jp
}

\begin{abstract}
Demand for positioning services such as object tracking systems and navigation systems is increasing. The current Wi-Fi positioning system uses the algorithm using mainly the signal strength. However, the signal strength is susceptible to multipath propagation, thus the ranging error generally large. The IEEE $802.11 \mathrm{mc}$ specifies the fine timing measurement (FTM) protocol based on round trip time (RTT). As of now, few commercial products support the FTM function. The objective of this letter is to evaluate the accuracy of the Wi-Fi FTM using the actual devices and to perform one-dimensional positioning by a simple algorithm. In the one-dimensional positioning, average positioning accuracy is $0.10 \mathrm{~m}$ in outdoor and $0.23 \mathrm{~m}$ in indoor.
\end{abstract}

Keywords: Wi-Fi, fine timing measurement (FTM), positioning

Classification: Wireless Communication Technologies

\section{References}

[1] IEEE Std 802.11-2016 (revision of IEEE Std 802.11-2012), "Part 11: Wireless LAN medium access control (MAC) and physical layer (PHY) specifications," IEEE Standard for Information technology — Telecommunications and information exchange between systems. Local and metropolitan area networks Specific requirements, 2016.

[2] M. Ibrahim, H. Liu, M. Jawahar, V. Nguyen, M. Gruteser, R. Howard, B. Yu, and F. Bai, "Verification: accuracy evaluation of WiFi fine time measurements on an open platform," Proc. 24th Annual International Conference on Mobile Computing and Networking (MobiCom '18), New Delhi, India, pp. 417-427, Oct. 2018. DOI: $10.1145 / 3241539.3241555$

[3] Google, Documentation for app developers, Wi-Fi location: ranging with RTT, https://developer.android.com/guide/topics/connectivity/wifi-rtt, accessed April $2,2020$.

\section{Introduction}

Location information is expected for several applications, such as object tracking systems and navigation systems. The global navigation satellite system (GNSS) is the most widely used in an outdoor environment, and whose main application is the navigation for such as cars and smartphones. However, the GNSS cannot apply to 
the indoor environment because there is no signal from satellites. A solution for an indoor environment is the use of wireless communication systems such as cellular systems, Wi-Fi, Bluetooth, and Zigbee. Most of the positioning algorithms are based on the received signal strength (RSS). However, signal strength is susceptible to multipath propagation, thus the ranging error generally large. There is the round-trip time (RTT) based measurement to overcome the inherent difficulties.

The IEEE802.11mc specifies the fine trimming measurement (FTM) based on RTT [1]. As of now, few commercial products support the FTM function, especially in Japan, there are very few products that have certificated the technical standard conformity certificate. As far as we searched, we find two kinds of devices that support the FTM. One is Intel Dual Band Wireless-AC 8260 NIC (Intel 8260) [2]. The other is Google Wi-Fi (product official name is Google Wifi) and Google Pixel 3 [3]. In the case of Intel 8260, RTT is obtained by the debug information; thus, RTT cannot be acquired by the general use. On the other hand, Android 9.0 Pie and above version support the FTM. Because of this situation in Japan, the objective of the letter is to clarify the accuracy of measurement distance using actual commercial devices and to clarify the accuracy of one-dimensional positioning by a simple algorithm.

\section{Fine timing measurement (FTM) protocol}

The IEEE $802.11 \mathrm{mc}$ specifies the FTM protocol to estimate the distance between two devices without association to a particular access point (AP). The distance is estimated by Time of Flight (ToF) using signal propagation. This protocol is used for the measurement of RTT based distance between two devices. In the FTM process, an initiator first transmits an FTM request to a responder corresponding to a device for which the distance is desired to be measured. After the initiator receives the response regarding the FTM request from the responder, the ranging process is started. The responder sends the FTM frame at time $t_{1}$ and waits for its acknowledgment (ACK). The initiator receives the FTM frame at time $t_{2}$ and responds with its $\mathrm{ACK}$ at time $t_{3}$. After that, the responder receives the $\mathrm{ACK}$ at time $t_{4}$ and sends the FTM frame, including $t_{1}$ and $t_{4}$. Through this process, the initiator has four types of times, $t_{1}, t_{2}, t_{3}$, and $t_{4}$. The difference time $\left(t_{4}-t_{1}\right)$, includes the processing time between the reception and transmission at the initiator $\left(t_{3}-t_{2}\right)$. The RTT excludes the processing time $\left(t_{3}-t_{2}\right)$; thus, it is $\left(t_{4}-t_{1}\right)-\left(t_{3}-t_{2}\right)$. The RTT based distance is calculated by $\left(\left(t_{4}-t_{1}\right)-\left(t_{3}-t_{2}\right)\right) \cdot c / 2$, where $c$ is the velocity of the radio signal.

\section{Experimental setup}

We use the following devices: Intel 8260 operated as an AP and a station (STA), Google Wi-Fi acted as an AP, and Google Pixel 3 (Pixel3) acted as an STA. The STAs act as the initiator, and the APs act as the responder. The limitation of the device is the frequency setting in the AP. The frequency settings of Intel 8260 operated as AP and Google Wi-Fi are $2.4 \mathrm{GHz}$ and $5 \mathrm{GHz}$, respectively. Besides, Intel 8260 as STA and Pixel3 support both $2.4 \mathrm{GHz}$ and $5 \mathrm{GHz}$, respectively. Note that the AP and STA are used, but the station need not associate with the AP. 
In this letter, two experiments in indoor and outdoor are conducted. The propagation between the AP and STA is a line-of-sight (LOS) environment. The objective of the first experiment is to clarify the accuracy of measurement distance using actual commercial devices. The combination of AP and STA is four types: AP (Intel 8260) and STA (Intel 8260), AP (Intel 8260) and STA (Pixel3), AP (Google Wi-Fi) and STA (Intel 8260), and AP (Google Wi-Fi) and STA (Pixel3). In these combinations, the antenna height of the AP and STA set the same height, and we measure the distance between the AP and STA three times. The height of the antenna is 1, 2, and $3 \mathrm{~m}$ for outdoor, and 1 and $2 \mathrm{~m}$ for indoor. The ceiling height limits the height of the antenna indoor. The first experiment also clarifies the accuracy difference in these combinations.

The second experiment is to clarify the accuracy of one-dimensional positioning by a simple algorithm in the case of the best accuracy combination in the first experiment. The difference from the first experiment, the height of STA is $1 \mathrm{~m}$. This is the height at which people usually use their smartphones. Besides, the AP is fixed at the height of $3 \mathrm{~m}$ for outdoor and $2 \mathrm{~m}$ for indoor.

\section{Experimental evaluation}

We evaluate the accuracy measurement through two experiments. The first experiment is to evaluate the distance accuracy. The second experiment is to evaluate the one-dimensional positioning accuracy. The measurement is conducted three times in each STA's position. We extract 200 data excluding data that could not be measured correctly, delete the outlier that is more than three scaled median absolute deviations (MAD) away from the median and use the mean of distance and RSS.

\subsection{Accuracy of measurement distance}

We experimented indoor and outdoor. Fig. 1(a) and (b) show the experimental environment outdoor. The antenna at the AP and STA is the same height. The number of measurement points is five; $5 \mathrm{~m}, 10 \mathrm{~m}, 15 \mathrm{~m}, 20 \mathrm{~m}$, and $25 \mathrm{~m}$ from the AP. The measurement results of distance and RSS in each devices' combination show Fig. 1(c) to (f). In all combinations of devices, it is evident that the RSS does not decrease with the actual distance and varies widely for each measurement. Therefore, it is clarified that the distance based on the RSS is low accuracy. In terms of the measured distance, the combination of AP (Intel 8260) and STA (Pixel3) has a great variation for each measurement compared with other combinations, and it is also evident that the measurement error is significant.

To further analysis of the measurement distance, we evaluate it by the mean absolute error (MAE). Fig. 2(a) shows the MAE in outdoor. The combination of AP (Google Wi-Fi) and STA (Pixel3) achieves the minimum MAE. In the $3 \mathrm{~m}$ height, the MAE is $0.26 \mathrm{~m}$. The other combinations, the MAE is over $1 \mathrm{~m}$. As with outdoor, in terms of indoor shown in Fig. 2(b), the combination of AP (Google Wi-Fi) and STA (Pixel3) achieves the minimum MAE. Unlike the outdoor environment, since the indoor environment is affected by multipath propagation, the MAE in indoor is larger than outdoor.

In the first experiment, it was found that the accuracy of measurement distance 


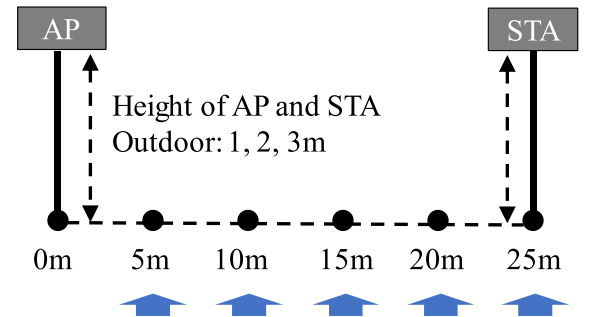

(a) Experimental environment
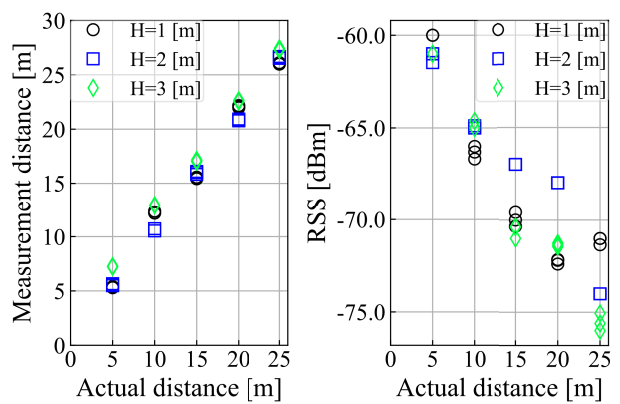

(c) Result in AP (Intel 8260) and STA (Intel 8260)
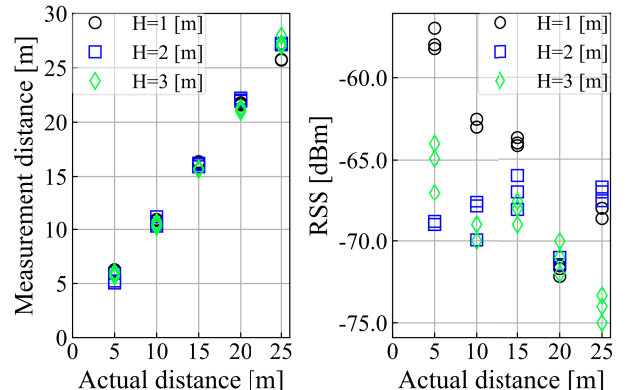

(e) Result in AP (Google Wi-Fi) and STA (Intel 8260)

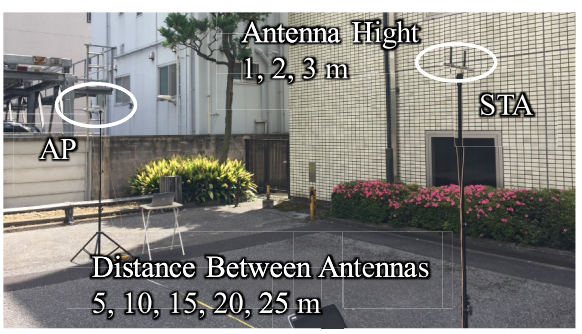

(b) Photo of outdoor environment
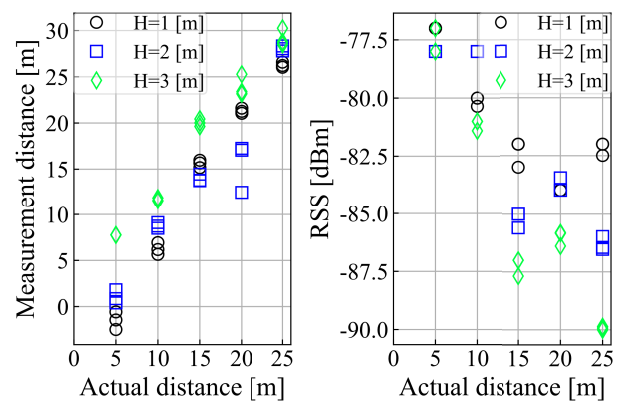

(d) Result in AP (Intel 8260) and STA (Pixel3)
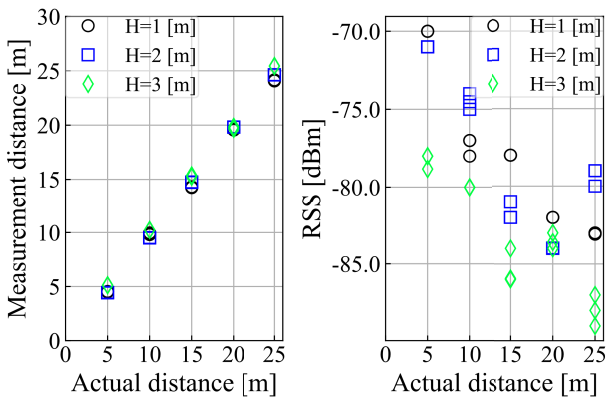

(f) Result in AP (Google Wi-Fi) and STA (Pixel3)

Fig. 1. Experimental environment and its results in outdoor.

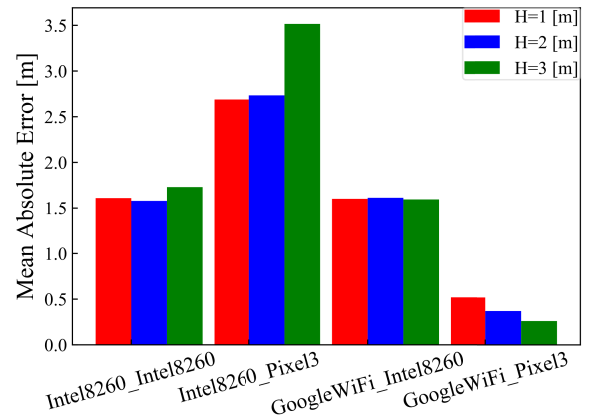

(a) Outdoor

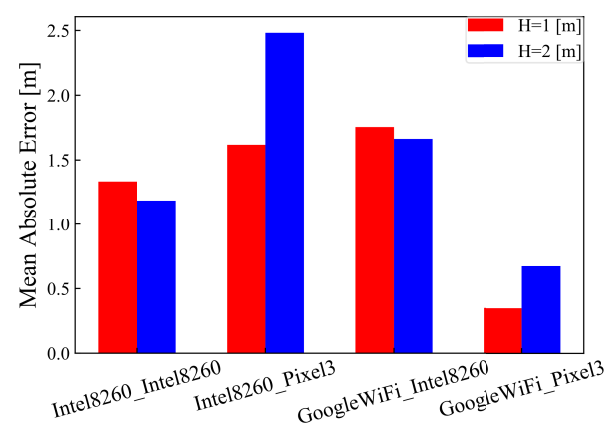

(b) Indoor

Fig. 2. Mean absolute error of measurement distance in outdoor and indoor. AP_STA denotes the combination of AP and STA.

depends on the device, and the best combination is AP (Google Wi-Fi) and STA (Pixel3).

\section{IEICE 2020}

DOI: 10.1587/comex.2020COL0001 Received April 3,2020 Accepted April 28, 2020 Publicized May 19, 2020 Copyedited December 1, 2020 


\subsection{Accuracy of one-dimensional positioning}

In the second experiment, the combination of AP (Google Wi-Fi) and STA (Pixel3) is used. An algorithm of one-dimensional positioning is straightforward. In Fig. 3(a), let $D, d_{1}$, and $d_{2}$ be the distance between AP1 and AP2, the measurement distance from AP1 and AP2, respectively. Assuming that the measurement distance, $d_{1}$, and $d_{2}$, includes the same error, the one-dimensional positioning point, $P$, is expressed as follows:

$$
P= \begin{cases}d_{1}+\left|\frac{\left(D-\left(d_{1}+d_{2}\right)\right.}{2}\right| & \text { if } D \geq d_{1}+d_{2} \\ d_{1}-\left|\frac{\left(D-\left(d_{1}+d_{2}\right)\right.}{2}\right| & \text { if } D<d_{1}+d_{2}\end{cases}
$$

We experimented indoor and outdoor. Fig. 3(b) shows the experimental environment. The number of measurement points is four; $5 \mathrm{~m}, 10 \mathrm{~m}, 15 \mathrm{~m}$, and $20 \mathrm{~m}$ from the AP1. We evaluate the accuracy of one-dimensional positioning by the MAE and compare the positioning point using both AP1 and AP2 with that using only AP1 and only AP2. Fig. 3(c) and (d) show the MEA in outdoor and indoor, respectively. In these figures, "Total" denotes the MAE in all points. Even if both AP1 and AP2 are used, the MAE may not be decreased at each point. In all points, MAE with the use of both AP1 and AP2 is decreased compared with the use of only AP1 or only AP2. In this case, average positioning accuracy is $0.10 \mathrm{~m}$ in outdoor and $0.23 \mathrm{~m}$ in indoor.

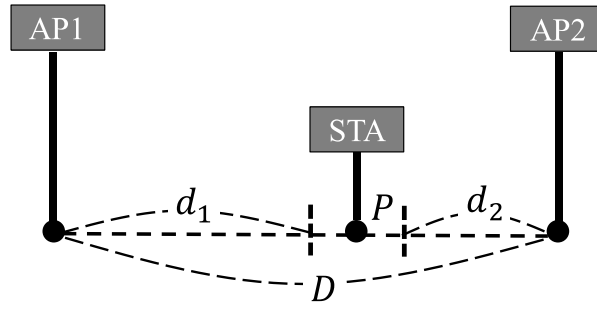

(a) Simple algorithm in case of $D \geq d_{1}+d_{2}$

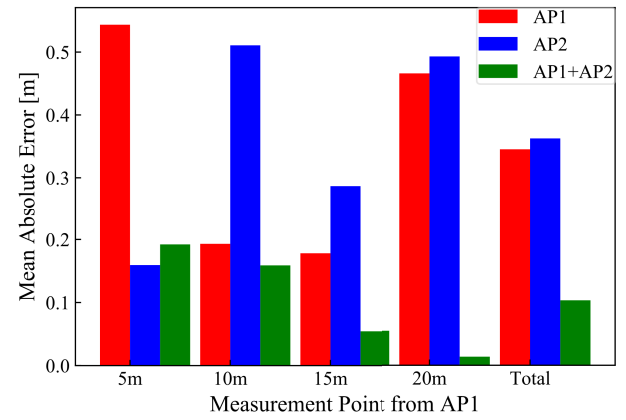

(c) MAE in outdoor

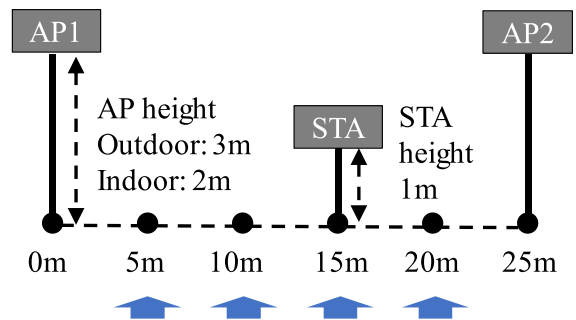

(b) Experimental environment

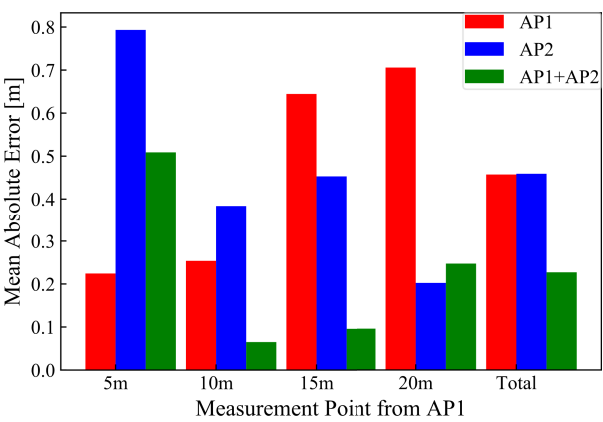

(d) MAE in indoor

Fig. 3. One-dimensional positioning algorithm, experimental environment, and mean absolute error of positioning in outdoor and indoor.

\section{Conclusion}

We have compared Wi-Fi FTM supported devices by the measurement distance. As of now, there is few Wi-Fi FTM supported devices, and it is necessary to clarify 
the measurement accuracy depends on devices. The experimental results show that the combination of Google Wi-Fi as AP and Google Pixel as STA achieves the best accuracy in indoor and outdoor. Besides, we have experimented with the one-dimensional positioning using two APs. We demonstrated that positioning accuracy is $0.10 \mathrm{~m}$ in outdoor and $0.23 \mathrm{~m}$ in indoor. Our future work is to consider a two-dimensional positioning algorithm. 